\title{
Serum Level of Anti-Hepatitis B Surface Antigen Among Students in Guilan, Iran
}

\author{
Yasaman Yaghobi ${ }^{1}$; Iraj Nikokar ${ }^{2,}$; Hadi Sedigh Ebrahim-Saraie ${ }^{3}$; Mojtaba Frahbakhsh ${ }^{2}$; \\ Mojtaba Safavi ${ }^{1}$; Robabeh Ansar ${ }^{1}$; Bahareh Fazli ${ }^{1}$ \\ ${ }^{1}$ Eastern Faculty of Nursing and Midwifery, Guilan University of Medical Sciences, Rasht, IR Iran \\ ${ }^{2}$ Laboratory of Microbiology and Immunology of Infectious Diseases, Para Medicine Faculty, Guilan University of Medical Sciences, Rasht, IR Iran \\ ${ }^{3}$ Department of Bacteriology and Virology, School of Medicine, Shiraz University of Medical Sciences, Shiraz, IR Iran \\ ${ }^{*}$ Corresponding author: Iraj Nikokar, Laboratory of Microbiology and Immunology of Infectious Diseases, Para Medicine Faculty, Guilan University of Medical Sciences, Rasht, IR Iran. \\ Tel: +98-1425237070, Fax: +98-1425237171, E-mail: Nikokariraj@yahoo.com; Nikokariraj@gums.ac.ir \\ Received: March 4, 2015; Revised: April 11, 2015; Accepted: April 21, 2015
}

\begin{abstract}
Background: Hepatitis B virus (HBV) is considered as a serious public health concern. The health care workers and medical students are at a higher risk of $\mathrm{HBV}$ infection than the general population through occupational exposure. Vaccination against HBV is considered an effective means of infection prevention.

Objectives: The aim of the present study was to determine anti-HBs titers among the medical students of Guilan University of Medical Sciences within the national HBV vaccination schedule.

Patients and Methods: This cross-sectional study comprised 209 medical students born between the years 1989 - 92 within a national vaccination gap of $\mathrm{HBV}$ and received $\mathrm{HBV}$ vaccine before age 18 years. The levels of anti-HBsAg $\geq 10 \mathrm{mIU} / \mathrm{mL}$ detected by ELISA were considered as protective against HBV.

Results: Of 209 students participated in this study, 11 (5.3\%) showed no response to HBV vaccination. The protective titers of $10-1000 \mathrm{mIU} /$ $\mathrm{mL}$, and greater than $1000 \mathrm{mIU} / \mathrm{mL}$ of anti-HBs were found in $126(60.2 \%)$ and $72(34.5 \%)$ of students, respectively.

Conclusions: The periodic monitoring of anti-HBs titers in medical students is important because of their awareness about the risk factors associated with HBV infection.
\end{abstract}

Keywords: Hepatitis B; Vaccines; Medical Student; Public Health

\section{Background}

Hepatitis B virus (HBV) is considered as a serious public health concern, since over 300 million people are estimated to be chronically infected with HBV around the world (1). The prevalence of HBV carriers shown varies ranging in different parts of the world from less than $1 \%$ to up $20 \%$ (2). The HBV chronic carriers' rate among Iranian reported up to $5 \%$ with varying ranges in different provinces (1). HBV infection leads to a range of devastating diseases from acute hepatitis to hepatocellular carcinoma (HCC) (3). An estimated, more than 30\% of chronically infected persons will ultimately develop a long term consequence of the disease, such as cirrhosis, end-stage liver disease, or HCC (3). The well-known routes of HBV transmission include perinatal, parenteral and sexual routes (4).

It is documented that health care workers and medical students are at a higher risk of HBV infection than general population through occupational exposure (5). Vaccination against HBV infection is considered as an effective means for prevention of infection. Therefore, as part of occupational safety vaccination is a necessity for all health care workers and medical students (6). HBV vaccination is part of the national routine immunization program for newborns in Iran since 1991 (7). However, because of a gap between vaccination coverage across the country all newborns were not vaccinated against HBV. Therefore, children born between the years 1989-1992 and were not vaccinated were later scheduled for HBV vaccination.

Upon HBV vaccination, anti-HBs appears in serum, which results in lifelong protection against $\mathrm{HBV}$ at titers up to $\geq 10 \mathrm{mIU} / \mathrm{mL}$ (8). This underlines the critical role of anti-HBs titer in people with occupational exposure to HBV infection. Such a regional study which determines anti-HBs titer in medical students could provide useful information in regard to the current situation and the basis for comparative researches.

\section{Objectives}

The aim of present study was to evaluate anti-HBs titer among the students of Guilan University of Medical Sciences born between 1989 and 1992 within a national HBV vaccination schedule.

Copyright (C) 2015, Health Policy Research Center. This is an open-access article distributed under the terms of the Creative Commons Attribution-NonCommercial 4.0 International License (http://creativecommons.org/licenses/by-nc/4.0/) which permits copy and redistribute the material just in noncommercial usages, provided the original work is properly cited. 


\section{Patients and Methods}

\subsection{Study Area and Design}

This cross-sectional study was carried out in 2012, and comprised 209 subjects including medical nursing, midwifery, and medical laboratory science students affiliated to Para medicine faculty, Guilan University of Medical Sciences, Rasht, Iran. Only students who received all 3 doses of $\mathrm{HBV}$ vaccine before age 18 years were included in the study. All students were generally healthy with no sign of acute or chronic illnesses. Any individual with history of anti-viral drug was excluded from the study. Informed written consent was obtained from each participant. This study was approved by Guilan University of Medical Science ethics committee.

\subsection{Diagnosis of Anti-HBsAg Level}

A 5 - $7 \mathrm{~mL}$ of venous blood sample was collected from each student to determine the serum level of anti-HBsAg using a commercial enzyme-linked immunosorbent assay (ELISA) (Dia.pro Diagnostic, Italy) according to manufacturer's protocol. The titers of anti-HBsAg $\geq 10 \mathrm{mIU} / \mathrm{mL}$ were considered as protective levels.

Statistical analyses were performed using SPSS software version 19. Results are presented as descriptive statistics in terms of relative frequency.

\section{Results}

Totally, 209 students participated in this study, including $62.3 \%$ females and $37.3 \%$ males with the age range between 18 and 22 years and the mean age $19 \pm 1$ SD. Generally, according to anti-HBs levels, all the participants who responded to vaccination were divided into four groups in terms of their anti-HBsAg levels (Table 1). More than two third of all vaccinated subjects with anti-HBs titer $\geq$ $10 \mathrm{mIU} / \mathrm{mL}$ were shown to be immune against HBV infection. Only in 11 (5.3\%) of the vaccinated participants had anti-HBs titer $<10 \mathrm{mIU} / \mathrm{mL}$ and did not respond to HBV vaccination.

Table 1. Distribution of Anti-HBsAg Levels in Studied Population

\begin{tabular}{lc}
\hline Anti-HBsAg Serum Levels, $\mathbf{m I U} / \mathbf{m L}$ & Values $^{\mathrm{a}}$ \\
\hline$<\mathbf{1 0}$ & $11(5.3)$ \\
$\mathbf{1 0 - 1 0 0}$ & $37(17.7)$ \\
$\mathbf{1 0 0 - 1 0 0 0}$ & $89(42.5)$ \\
$>\mathbf{1 0 0 0}$ & $72(34.5)$ \\
\hline
\end{tabular}

${ }^{\mathrm{a}}$ Values are presented as No. (\%).

\section{Discussion}

The present study demonstrated the levels of anti-HBs in medical students born between 1989 and 1992 who received three doses of HBV vaccine. Medical students are at greater risk of HBV infection than other healthcare staff and are required to be vaccinated against $\mathrm{HBV}$, because of their occupational risk and lacking experience during their professional training (5).

Symptomatic hepatitis B is very uncommon in persons who received HBV vaccine and have anti-HBs titers $\geq 10$ $\mathrm{mIU} / \mathrm{mL}$, although there are evidences on loss of detectable anti-HBs titers in up to half of these persons few years after immunization (1). This is especially observed in adolescence vaccinated against HBV in the neonatal period (2), which must therefore be monitored for antiHBs titers.

Our results showed that $94.7 \%$ of the medical students had antibody level greater than $10 \mathrm{mIU} / \mathrm{mL}$, which is considered as highly efficient anti-HBs titers to prevent HBV infection. It has been stated that starting the initial vaccination series later in infancy may result in better level of anti- HBsAg in future (1). Several studies conducted on healthcare staffs in Iran assessed the immune response against HBV vaccine. Alavian et al. (9) from faculty of dentistry, Tehran reported anti-HBs titers 10 - $99 \mathrm{mIU} / \mathrm{mL}$ in $17.8 \%$ of 230 participants and $69.1 \%$ of the subjects studied had an anti-HBsAg titer of more than $100 \mathrm{mIU} / \mathrm{mL}$, accounting for an overall 86.9\% immunity level. In another study in Tehran by Zamani et al. (10) among 151 healthcare workers $68.2 \%$ of subjects had anti-HBs titer $>10 \mathrm{mIU} / \mathrm{mL}$. Previous study from Guilan province carried out by Mansour Ghanaei et al. (11) showed 95.1\% of students had a protective level of anti-HBsAb, and stated that it was the maximum response rate in Iran. Moreover, Zehni et al. (12) showed the protective HBV antibody titer among $86 \%$ of nursing and midwifery faculty members of Kurdistan University of Medical Sciences (Sanandaj, Iran) in 2013. In a study by Hegde et al. (13), from 110 students of a dental college in India, 7.5\% of subjects had less than $100 \mathrm{mIU} / \mathrm{mL}$ anti-HBs level and 92.5\% had more than $100 \mathrm{mIU} / \mathrm{mL}$ anti-HBs level. Based on the findings of a meta-analysis study by Tazhibi et al. (14) regarding efficacy of protective level of anti- HBsAg titer our results are closest to those of general population, since they found the efficiency of hepatitis B vaccine in Iranian general population to be more than $80 \%$.

In the present study, no immune response to vaccination was rare, since only 5.3\% participants had anti-HBs titer $<10 \mathrm{mIU} / \mathrm{mL}$. A series of studies carried out in Iran reported different rates of non-respond anti-HBs titer ranging from very low in 3.3\% by Mohsenzade et al. (8) to relatively notable frequency in $17.2 \%$ of the subjects studied by Zamani et al. (10). However, non-protective level of anti-HBsAg among $5.3 \%$ of our participants may be due to a reduction in the level of anti-HBsAg over time and approximately five years after vaccination $(1,7)$.

It has been stated that the administration of late booster doses of HBV vaccine are not necessary in those who have detectable anti-HBs titers. In this context, routine anti-HBs titers monitoring is necessary, since HBV is one the most important infectious occupational risks for the health care workers $(1,5)$. Iran is a low HBV endemic area 
and therefore, special attention is required to determine the efficiency of vaccination coverage for $\operatorname{HBV}(2)$.

Our finding showed that $77 \%$ of the participants had anti-HBs titers greater than $100 \mathrm{mIU} / \mathrm{mL}$ and because of medical students are at higher risk of HBV exposure, this rate of coverage seems to be insufficient. Therefore, with respect to the vulnerability of the medical students to $\mathrm{HBV}$, it is important to carry out routine assessment of anti-HBs titers following anti-HBV vaccination in medical students and health care workers.

\section{Acknowledgements}

The authors would like to thank all the participants for their sincere cooperation in this study.

\section{Funding/Support}

Guilan University of Medical Sciences, Rasht, IR Iran.

\section{References}

1. Kazemi A, Koosha A, Rafizadeh B, Mousavinasab N, Mahram M. Serum level of anti-hepatitis B surface antigen 6-8 years after hepatitis B vaccination at birth. East Mediterr Health J. 2008;14(4):960-5.

2. Aghakhani A, Banifazl M, Izadi N, McFarland W, Sofian M, Khadem-Sadegh A, et al. Persistence of antibody to hepatitis B surface antigen among vaccinated children in a low hepatitis B virus endemic area. World J Pediatr. 2011;7(4):358-60.

3. Liang TJ. Hepatitis B: the virus and disease. Hepatology. 2009;49(5 Suppl):S13-21.

4. Vinodhkumaradithyaa A, Srinivasan M, Sankarasubramanian R, Uma A, Ananthalakshmi I, Thirumalaikolundusubramanian P, et al. Hepatitis B vaccination among medical students. Indian J Community Med. 2008;33(1):67-8.
5. Noubiap JJ, Nansseu JR, Kengne KK, Tchokfe Ndoula S, Agyingi LA Occupational exposure to blood, hepatitis B vaccine knowledge and uptake among medical students in Cameroon. BMC Med Educ. 2013;13:148.

6. Hadi N, Hadi N. Assessment of anti-HBs antigen in 6-to 9-year-old children routinely vaccinated via vaccination program in Iran. Med Princ Pract. 2007;16(4):306-9.

7. Jafarzadeh A, Sajjadi SMA. Persistence of anti-HBs antibodies in healthy Iranian children vaccinated with recombinant hepatitis B vaccine and response to a booster dose. Acta Med Iran. 2005;43(2):79-84

8. Mohsenzade M, Pirouzi A, Jafari M, Azadi M, Raeiszadeh-Jahromi S, Afkari R. Study on the Serum Titer of Anti-HBs Antibody of the Vaccinated Staffs of a Hospital in Three Consecutive Years. 2. 2013;15(12):23-7.

9. Alavian SM, Mahboobi N, Mahboobi N. Anti-HBs antibody status and some of its associated factors in dental health care workers in Tehran University of Medical Sciences: Anti-HBs Ab and associated factors in dental society. Hepat Mon. 2011;11(2):99-102.

10. Zamani F, Fallahian F, Hashemi F, Shamsaei Z, Alavian SM. Immune response to hepatitis $\mathrm{B}$ vaccine in health-care workers. Saudi J Kidney Dis Transpl. 2011;22(1):179-84.

11. Mansour Ghanaei F, Fallah MS, Jafarshad R, Joukar F, Arami M, Ale-Esmaeil A, et al. The Immunologic Response to anti-hepatitis B vaccination among medical students of Guilan University of Medical Sciences, Guilan, Iran. Hepat Mon. 2006;2006(2, Spring):63-6.

12. Zehni K, Rokhzadi MZ, Mohmodi SH, Ashjardalan A. Vaccination and Immunity Status against Hepatitis B among Students of Nursing and Midwifery Faculty of Kurdistan University of Medical Sciences in 2013. Life Sci J. 2013;10(7s):23-8.

13. Hegde SH, Pal S, Shishir Shetty R, Ajila V, Babu S. Serum antibody analysis following Hepatitis B vaccination for occupational risk assignment among dental students. Nitte Univ J Health Sci. 2014;4(1):52-6.

14. Tazhibi M, Hajivandi A, Tafti AD, Fallahzadeh H. The efficacy of hepatitis B vaccine in Iranian population: A systematic review and meta-analysis. J Educ Health Promot. 2014;3:53. 